\section{THE RATIONALE FOR A REDEFINITION OF VISUAL ART BASED ON NEUROAESTHETIC PRINCIPLES}

Eugen Bogdan Petcu, Griffith University School of Medicine Gold Coast Campus, Griffith University, QLD 4222, Australia. Email: <e.petcu@griffith.edu.au>.

See $<$ www.mitpressjournals.org/toc/leon/51/1 $>$ for supplemental files associated with this issue.

Submitted: 8 June 2017

\section{Abstract}

Recent investigational studies have indicated that fronto-orbital, temporal and parietal lobes have a decisive role in artistic creation and personal identification of "beauty" in painting. Moreover, visual artistic work and preferences could be modified by central nervous system diseases or external stimuli such as transcranial magnetic stimulation. Both creation and preferences would depend on prior art education and sociocultural norms. However, the superior activity of the brain remains of paramount importance in production and evaluation of paintings regardless of the style, representational or abstract. Therefore, redefinition of art by neuroaesthetic principles will create a better communication between the public and the artists.

Visual artistic creation and its aesthetic evaluation are intrinsically linked to human evolution and socio-cultural context. In fact, visual art represents a superior form of human communication solely dependent on the central nervous system's (CNS) integrated activity. There are a multitude of various styles or representations and people have different preferences in terms of classical or abstract productions. An artist's style changes over time or after diseases affecting the CNS processing and integration. Therefore, the development of a new artistic style or a change in preference for a specific type of art seen in nonartists represents an "evolution" within a different pattern of communication determined by a new level of CNS activation. In this setting, neuroaesthetics represents a new field at the interface between art and medical sciences that aims to explain the neural mechanisms of artistic creation and aesthetic appreciation. Neuroaesthetics relies on new investigational methods for evaluation of central nervous system functionality, such as magnetic resonance imaging (MRI) or electroencephalography (EEG) [1][2]. Neuroaesthetic studies have been dramatically improved by quantitative methods such as the implementation of the Chatterjee's Assessment of Art Attributes, which is based on the evaluation on a 5-point Likert scale of several conceptual and formal attributes of 24 masterpieces of the Western art historical canon, signed by various artists from Holbein and Van Eyck to Picasso, Rothko, De Kooning and Dalí. Conceptual art attributes evaluated by this method include accuracy, abstraction-realism, emotions and symbolism, while the formal characteristics evaluated are represented by color saturation and temperature, stroke, depth, balance and complexity [3]. Most of the studies investigating the neurobiology of artistic creation and evaluation have indicated that both may be modulated by centers located in the frontal, parietal and temporal lobes. We can consider as the "classical" benchmark the MRI study conducted by Kawabata and Zeki in healthy volunteers, which indicated that the orbito-frontal area of the brain is activated differently when evaluating copies of paintings encompassing various themes: portraits, landscapes and modern representations [4]. For the first time ever, the authors of this study separate the paintings into "beautiful," "ugly" and "neutral" based on the activity recorded in the orbito-frontal CNS region, which is in fact a specialized area related to pleasure and reward mechanisms [5]. Remarkably, evaluation of abstract modern art, which often is considered hermetical, also determines CNS changes identified by EEG as $\mathrm{Mu}$ rhythm abolition. This is interesting as the Mu rhythm represents a normal pattern of an EEG. While the exact significance of this is not fully understood, it indicates that "enjoyment" of modern abstract art implies electric visual processing substantiated by EEG changes [6].

Other clinical studies have revealed that aesthetic evaluation is associated not only with activation of fronto-medial cortex but also that it may depend on cognitive processes related to various social norms implying a complex neural cognitive network association $[7,8]$. From the anatomo-morphological perspective, some authors have proved that classification into "beauty" and "ugly" visual artworks requires other CNS regions such as left temporal and temporo-parietal regions. The personal "labeling" of "beauty" requires also the activation of the left intraparietal sulcus, which is involved in symmetry appreciation, and the amygdala, a specific area within the temporal lobe that controls emotions and memory processing $[9,10]$. However, other authors have indicated that CNS processing of art's style follows the evaluation of its content. Healthy subjects with no formal art training were exposed to an equal number of high-quality photos of landscapes and portraits by Ernst Ludwig Kirchner and Paul Cezanne. In parallel, electroencephalographic (EEG) recordings indicated that the artistic style is perceived at 40-90 milliseconds later than the actual content of the artwork [11].

However, CNS activation induced by art appreciation may be different in those with art education. When a group of students was exposed to images of masterpieces from various museums and also to abstract but obscure digital compositions created by computer, the students' neural responses were paradoxically different. The activation of frontal and orbito-frontal regions was higher when subjects evaluated consecrated artworks displayed in museums, suggesting a bias explained most likely by artistic education and previous exposure and/or learning with activation of the entorhinal-temporal regions [12]. Intriguingly, some authors have suggested that personal preferences for a certain type of art may be altered by stimulation of specific brain regions. More specifically, application of transcranial magnetic stimulation (TMS) of the left dorsolateral prefrontal cortex induces a significant decrease for the preferred type of art, abstract or representational [13]. A subsequent study indicated that TMS applied over the lateral occipital cortex does not interfere with evaluation of abstract art but significantly impairs appreciation for classical representational works [14].

Although this would suggest that aesthetic preferences could be modulated by activation of specific brain regions, including visual recognition processing, at the present time the morphological pathways and the associated molecular factors related to artistic creation and appreciation are poorly understood. Meanwhile, a significant wealth of information regarding the neurobiology of artistic appraisal as well as conception and creation has been derived from studies conducted on patients including artists with CNS lesions. Bromberger et al. have revealed in a clinical study mapping by functional MRI the right cerebral hemisphere that in patients with lesions located at this level, regardless of the specific type, the ability of the patient to evaluate the accuracy and style is impaired [15]. Also, morphological changes in the temporal and frontal parietal lobes determine anomalies related to the patient's ability to identify animacy, realism-abstractness and symbolic representation [16]. 
A recent comprehensive evaluation of artists who had suffered a cerebral stroke indicates that, often after the acute event, the artists would develop an alternative mode of creation, with or without recovery of their style. From a clinical neurological perspective, changing of an artist's style after stroke represents an adaptive mechanism allowing communication of the patients with their environment [17]. The artwork of these artists is characterized initially by a unilateral nonrepresentation as a result of a unilateral neurological neglect determined by focal stroke destruction on the same side. In these patient-artists the aesthetic aspect of the artistic creation is less important than the actual communication through art with the public and their peers. Moreover, the communication through artistic work is very important for those artists with severe degenerating CNS conditions such as Alzheimer's disease. The case of Willem de Kooning is already well-known. In his early career, the artist embraced a modern abstract expressionist style that changed dramatically during the last few years of his life when he was diagnosed with Alzheimer's. However, his paintings continued to be appreciated both by the peers and public. Espinel describes in de Kooning's case, a syndrome of "Creating in the Midst of Dementia" [18]. Communication through art is also the last remaining method in some reported cases of patients with fronto-temporal dementia, a degenerative condition characterized by severe atrophy located at the level of frontal and temporal lobes. Apart from severe dementia, these non-artistically trained patients develop a strong interest for painting. It might be possible that atrophyinduced destruction or an altered inhibition/activation of some fronto-temporal centers could explain this artistic behavior.

However, their works are characterized by a definite obsession with certain subjects, colors and forms, with a striking interest for minute details. We could speculate that compulsive drawing of the same topic seen in these patients could be related to a possible activation of a rewarding mechanism in the superior brain centers. Characteristically, the artistic abilities of these patients reach a peak, but subsequently they vanish as the disease progresses [19]. Therefore, we could label these patients as suffering from a "Temporary Artistic Syndrome," which nevertheless conveys a strong artistic message to the public.

Overall, artistic creation and appreciation is associated with changes in superior centers located in the CNS including the fronto-orbital, temporal and parietal lobes. The study of art in those with dementia indicates that visual art creation is indeed a method of communication substantiated by specific activity in the brain recorded by functional methods. While certain art preferences are related to prior art education, these may change after CNS disease and could be modified by TMS. The artistic creation itself seems to be determined by activation of superior CNS centers, including areas related to visual processing, memory, superior emotions and rewarding mechanisms. Since both representational and abstract paintings determine CNS activation, we could redefine visual art as any creation that simply stimulates specific regions within CNS, allowing us to label a drawing or painting not necessarily according to its style but as "ugly," "beautiful" or "indifferent." Ultimately, neuroaesthetics represents a method that could help both the public and the artists to better understand the meaning of artistic creation by redefining its rationale and limits.

\section{References}

1. G.C. Cupchik et al., "Viewing Artworks: Contributions of Cognitive Control and Perceptual Facilitation to Aesthetic Experience," Brain Cognition 70, No. 1, pp. 84--91 (June 2009). doi: 10.1016/j.bandc.2009.01.003.

2. T. Jacobsen et al., "Brain Correlates of Aesthetic Judgment of Beauty", Neuroimage 29, No. 1, pp. 276--285 (1 January 2006).

3. A. Chatterjee et al., "The Assessment of Art Attributes," Empirical Studies of the Arts 28, No. 2, pp. 207--222 (First published 11August 2010) doi: 10.2190/EM.28.2.

4. H. Kawabata, S. Zeki, "Neural Correlates of Beauty," Journal of Neurophysiology 91, No.4, pp. 1699--1705 (April 2004).

5. H. Kawabata, S. Zeki [4].

6. M.A. Umilta et al., "Abstract Art and Cortical Motor Activation: An EEG Study," Frontiers in Human Neuroscience 6 (16 Nov 2012) p. 311. doi: 10.3389/fnhum.2012.00311.

7. Jacobsen et al. [2].

8. U. Kirk et al., "Modulation of Aesthetic Value by Semantic Context: An fMri Study," Neuroimage 44, No. 3, pp. 1125--1132 (1 February 2009). doi: 10.1016/j.neuroimage.2008.10.009.

9. K. Amunts et al., "Cytoarchitectonic Mapping of the Human Amygdala, Hippocampal Region and Entorhinal Cortex: Intersubject Variability and Probability Maps," Anatomy and Embryology) 210, Nos. 5-6, pp. 343-352 (December 2005).

10. Jacobsen et al. [2].

11. M.D. Augustin et al., "The Neural Time Course of Art Perception: An ERP Study on the Processing of Style Versus Content in Art," Neuropsychologia 49 No. 7, pp. 2071-2081 (June 2011). doi: 10.1016/j.neuropsychologia.2011.03.038.

12. Kirk [8].

13. Z. Cattaneo et al., "The Role of Prefrontal and Parietal Cortices in Esthetic Appreciation of Representational and Abstract Art: A TMS Study," Neuroimage, No. 99 (1 October 2014) pp. 99:443--50. doi:

10.1016/j.neuroimage.2014.05.037.

14. Z. Cattaneo et al., "The Role of the Lateral Occipital Cortex in Aesthetic Appreciation of Representational and Abstract Paintings: A TMS Study," Brain Cognition 95 (April 2015) pp. 44-53. doi: 10.1016/j.bandc.2015.01.008.

15. B. Bromberger et al., "The Right Hemisphere in Esthetic Perception," Frontiers in Human Neuroscience 5 (14 October 2011) p. 109. doi: 10.3389/fnhum.2011.00109.

16. Bromberger et al. [15].

17. E.B. Petcu et al., "Artistic Skills Recovery and Compensation in Visual Artists after Stroke," Frontiers in Neurology 7 (13 May 2016) p. 76. doi: 10.3389/fneur.2016.00076.

18. C.H. Espinel, "Memory and the Creation of Art: The Syndrome, as in De Kooning, of 'Creating in the Midst of Dementia,'" Frontiers of Neurology and Neuroscience 22 (2007) pp. 150-68.

19. B.L. Miller et al., "Emergence of Artistic Talent in Frontotemporal Dementia," Neurology 51, No. 4, pp. 978-982 (October 1998). 